Hiroshi Mitsumoto, MD DSc

Peter L. Nagy, MD, PhD Chris Gennings, $\mathrm{PhD}$ Jennifer Murphy, PhD Howard Andrews, PhD Raymond Goetz, PhD Mary Kay Floeter, MD, $\mathrm{PhD}$

Jonathan Hupf, BA Jessica Singleton, BA Richard J. Barohn, MD Sharon Nations, MD Christen Shoesmith, MD Edward Kasarskis, MD, $\mathrm{PhD}$

Pam Factor-Litvak, PhD For the PLS COSMOS Study Group

Correspondence to Dr. Mitsumoto: hm264@cumc.columbia.edu

Supplemental data at Neurology.org/ng

\section{Phenotypic and molecular analyses of primary lateral sclerosis} OPEN

\section{ABSTRACT}

Objective: To understand phenotypic and molecular characteristics of patients with clinically "definite" primary lateral sclerosis (PLS) in a prospective study.

Methods: Six sites enrolled 41 patients who had pure upper motor neuron dysfunction, bulbar symptoms, a normal EMG done within 12 months of enrollment, and onset of symptoms $\geq 5$ years before enrollment. For phenotypic analyses, 27 demographic, clinical, and cognitive variables were analyzed using the k-means clustering method. For molecular studies, 34 available DNA samples were tested for the C9ORF72 expansion, and exome sequencing was performed to exclude other neurologic diseases with known genetic cause.

Results: K-means clustering using the 25 patients with complete datasets suggested that patients with PLS can be classified into 2 groups based on clinical variables, namely dysphagia, objective bulbar signs, and urinary urgency. Secondary analyses performed in all 41 patients and including only variables with complete data corroborated the results from the primary analysis. We found no evidence that neurocognitive variables are important in classifying patients with PLS. Molecular studies identified C9ORF72 expansion in one patient. Well-characterized pathogenic mutations were identified in SPG7, DCTN1, and PARK2. Most cases showed no known relevant mutations.

Conclusions: Cluster analyses based on clinical variables indicated at least 2 subgroups of clinically "definite" PLS. Molecular analyses further identified 4 cases with mutations associated with amyotrophic lateral sclerosis, Parkinson disease, and possibly hereditary spastic paraplegia. Phenotypic and molecular characterization is the first step in investigating biological clues toward the definition of PLS. Further studies with larger numbers of patients are essential. Neurol Genet 2015;1:e3; doi: 10.1212/01.NXG.0000464294.88607.dd

\section{GLOSSARY}

ALS = amyotrophic lateral sclerosis; ALS-CBS = Amyotrophic Lateral Sclerosis-Cognitive Behavioral Screen; COWAT = Controlled Oral Word Association Test; HSP = hereditary spastic paraplegia; IRB = Institutional Review Board; $\mathbf{L M N}=$ lower motor neuron; LPGM = Laboratory of Personalized Genomic Medicine; MND = motor neuron disease; PD = Parkinson disease; $\mathbf{P L S}=$ primary lateral sclerosis; $\mathbf{S N P}$ = single nucleotide polymorphism; UMN = upper motor neuron.

Primary lateral sclerosis (PLS) is considered the rarest motor neuron disease (MND). It is clinically characterized by isolated pure upper motor neuron (UMN) dysfunction. Thus, the absence of lower motor neuron (LMN) involvement distinguishes PLS from amyotrophic lateral sclerosis (ALS). ${ }^{1}$ The diagnosis of PLS is also based on excluding all definable diseases, such as multiple sclerosis, myelopathy, metabolic diseases, and hereditary spastic paraplegia (HSP). In addition, after a few years of observation, some patients with suspected PLS develop features of LMN

From the Department of Neurology (H.M., J.H., J.S.), Eleanor and Lou Gehrig MDA/ALS Research Center, Columbia University Medical Center (CUMC), New York, NY; Department of Pathology and Cell Biology (P.L.N.), Personalized Genomic Medicine Laboratory, CUMC, New York, NY; Department of Biostatistics (C.G.), Virginia Commonwealth University, Richmond, VA; Department of Neurology (J.M.), University of California, San Francisco, CA; Departments of Biostatistics and Psychiatry (H.A., R.G.), Mailman School of Medicine, CUMC, New York, NY; Clinical Neuroscience Program (M.K.F.), NINDS, NIH, Bethesda, MD; Department of Neurology (R.J.B.), University of Kansas, Lawrence, KS; Department of Neurology (S.N.), University of Texas Southwestern Medical Center, Dallas, TX; Department of Neurology (C.S.), Western University, London, Ontario, Canada; Department of Neurology (E.K.), University of Kentucky, Lexington, KY; and Department of Epidemiology (P.F.-L), Mailman School of Public Health, CUMC, New York, NY.

PLS Study COSMOS Group coinvestigators are listed at Neurology.org/ng.

Funding information and disclosures are provided at the end of the article. Go to Neurology.org/ng for full disclosure forms. The Article Processing Charge was paid by Columbia University.

This is an open access article distributed under the terms of the Creative Commons Attribution-Noncommercial No Derivative 3.0 License, which permits downloading and sharing the work provided it is properly cited. The work cannot be changed in any way or used commercially. 
dysfunction. ${ }^{2}$ Consequently, the diagnostic criteria for PLS have been extended from the originally recommended 3 years without LMN involvement to 4 years. $^{3-5}$ Although patients with PLS have markedly impaired motor function, PLS, unlike ALS, is not life-threatening. PLS is suspected to be a heterogeneous disease. ${ }^{6,7}$ Because of its rarity, no prospective studies have analyzed the clinical and molecular characteristics of PLS. However, such investigations are an essential first step to clearly defining PLS and understanding the mechanisms underlying the disease. Here, we report novel analyses of the clinical phenotype and genetic markers of PLS.

METHODS Standard protocol approvals, registrations, and patient consents. The study protocol was approved by the Institutional Review Board (IRB) of Columbia University (Protocol Numbers: AAAE1115 and AAAE4850) and the individual IRBs of all participating sites.

Eligible patients were prospectively enrolled in this study, which is a parallel study to ALS Multicenter Cohort Study of Oxidative Stress (ALS COSMOS) (NIEHS, R01ES016348).

These 2 studies are nearly identical in structure and methodology, ${ }^{6}$ except for eligibility criteria, follow-up visit frequency, and number of participating sites. ${ }^{8}$

We defined clinically "definite" PLS when patients had (1) pure UMN dysfunction for at least 5 years following symptom onset based on subjective functional impairment ${ }^{2,3}$; (2) a normal EMG done within 12 months of enrollment (minimum changes in only one muscle were permitted) $)^{2,4}$; and (3) normal brain and spinal cord neuroimaging with allowance for increased signal intensity in the pyramidal tracts at the posterior internal capsules. ${ }^{9}$ Exclusion criteria included patients in whom only the legs were affected, patients with known HSP, patients with a history of MND in immediate family, or those with other active neurologic and unstable medical diseases. The absence of bulbar symptoms, such as dysarthria, dysphagia, or pseudobulbar affect, although preferred, was not exclusionary. ${ }^{10}$

Biospecimens (blood, DNA, urine, and skin biopsies) were collected, processed, and stored using appropriate methods. The patients had extensive clinical examinations and cognitive testing and had well-validated structured interviews as fully described in ALS COSMOS. ${ }^{8}$

Phenotype cluster analyses. We collected data on 27 demographic and clinical variables for all 41 enrolled patients (table e- 1 at Neurology.org/ng). Primary analyses were conducted on the 25 patients with complete data; secondary analyses were conducted on all 41 (table 1). Of the 27 potential clinical indicators, 5 (memory impairment, car sickness, stuttering, difficulty spelling, and predominantly unilateral symptoms) were present in no more than 1 patient and therefore excluded from the analysis. Verbal fluency was measured with the Written Verbal Fluency Test or the Controlled Oral Word Association Test (COWAT) with F, $A$, and S, which consisted of a combination of scores from the 3 subscales. Clinical verbal impairment was defined as either a Fluency Test index score of at least 19 or a COWAT score at least 2 SDs below the mean, adjusted for sex, age, and education level. ${ }^{11}$ Finally, before conducting the cluster analysis, we standardized all variables, continuous and binary, to a mean of 0 and an SD of 1 to ensure that they had equal weight in the analysis. ${ }^{12}$ A sensitivity analysis in which we scaled only the continuous variables and left the binary variables as 0 or 1 showed no meaningful difference.

In a secondary analysis, we restricted the variables to those with data available from all 41 patients, i.e., age, sex, disease duration, and the 15 binary clinical variables. A cluster analysis was performed following the method described above. Data analyses were conducted using SAS version 9.2 (SAS Institute, Cary, NC) and R version 3.0.1.

K-means clustering. In preliminary analyses, we examined the plot of the within-groups sum of squares over a range of 2-15 possible clusters. Because we did not observe a "bend" in the plot (indicating the optimal number of clusters) and this was an exploratory analysis, we opted for 2 clusters in order to be conservative and for ease of interpretation (figure 1).

We identified the 2 clusters using k-means with Euclidean distance. Variables associated with the clustering were identified in 2 steps. First, a model-building strategy using least absolute shrinkage and selection operator (Lasso) identified potentially important variables. ${ }^{13}$ These were included in a logistic regression model. A Wald test for significance of parameters in the logistic model was used to identify important clustering variables.

Genetic analysis. All testing on the available 34 patient samples was performed in the Laboratory of Personalized Genomic Medicine (LPGM) at Columbia University.

C9ORF72 expansion testing. The presence of expanded repeats was determined using the method of Renton et al. ${ }^{14}$

Sequencing and bioinformatics. Sequence capture for highthroughput sequencing was performed using Illumina TruSeq exome capture reagents, and 100-bp paired-end sequencing was performed on an Illumina HiSeq 2500 sequencer. Next-generation sequencing data were mapped and variants were called using NextGENe (SoftGenetics, State College, PA). ${ }^{15}$ Variant filtering and comparative analysis were performed using the single nucleotide polymorphism (SNP) catcher software developed by the LPGM. This software relies on allele frequency and functional prediction data from multiple publicly available databases, including ClinVar, ${ }^{16}$ 1000 Genomes Project, ${ }^{17}$ Exome Variant Server, ${ }^{18}$ and MSV3D. ${ }^{19,20}$ Pathogenic mutations were confirmed by Sanger sequencing.

RESULTS All 41 patients had clinically typical PLS. Six patients had no dysarthria or dysphagia, and another 6 had the minimum EMG changes that were permitted for enrollment (table e-1). All patients were alive at the time of report, except 3 who died unexpectedly (patient 7 had a sudden death during sleep, patient 11 had a fall resulting in death, and patient 12 had sepsis resulting in respiratory and cardiac failure). Patient 4 had abnormal CSF (protein of $118 \mathrm{mg} / \mathrm{dL}$ and 8 cells) due to a traumatic spinal tap.

Table 1 compares sociodemographic, clinical, and cognitive variables between all patients $(n=41)$ and those patients with complete data $(n=25)$. Generally, the data were similar across the 2 datasets, with a slightly lower percentage of women and patients with dysarthria, dysphagia, and objective bulbar signs in patients with complete data.

Using Euclidean distance measures, we constructed a dendrogram to visualize the similarities in 
Table 1 Sociodemographic, clinical, and cognitive data for patients with PLS

\begin{tabular}{|c|c|c|}
\hline Characteristic & All patients $(\mathrm{N}=41)$ & $\begin{array}{l}\text { Patients wit } \\
\text { data ( } N=2\end{array}$ \\
\hline \multicolumn{3}{|l|}{ Demographic variables } \\
\hline Age, y & $60(9.1)$ & $60(8.0)$ \\
\hline Disease duration at baseline, $y$ & $7.4(2.0)$ & $7.1(2.1)$ \\
\hline Sex, \% female & 46 & 40 \\
\hline \multicolumn{3}{|l|}{ Clinical signs and evaluations, \% } \\
\hline Dysarthria & 83 & 76 \\
\hline Dysphagia & 46 & 36 \\
\hline Objective bulbar signs & 44 & 36 \\
\hline Minimum EMG changes & 15 & 16 \\
\hline Urinary urgency & 39 & 36 \\
\hline Bowel urgency & 5 & 4 \\
\hline Weight loss & 7 & 12 \\
\hline Personality/cognitive changes & 10 & 4 \\
\hline Sensory impairment & 12 & 16 \\
\hline No SSEP from legs & 2 & 4 \\
\hline \multicolumn{3}{|l|}{ Cognitive/behavioral test scores } \\
\hline \multirow[t]{2}{*}{ Mini-Mental State Examination } & $28.8(1.6)$ & $28.6(1.8)$ \\
\hline & $N=38^{a}$ & \\
\hline \multirow[t]{2}{*}{ ALS-CBS cognitive } & $14.4(2.9)$ & $14.1(3.1)$ \\
\hline & $N=37^{a}$ & \\
\hline \multirow[t]{2}{*}{ ALS-CBS behavioral } & $9.6(8.4)$ & $9.0(8.0)$ \\
\hline & $N=32^{a}$ & \\
\hline Verbal impairment, \% ${ }^{\mathrm{b}}$ & 85 & 80 \\
\hline \multirow[t]{2}{*}{ CNS-LS } & $15.3(5.3)$ & $14.8(4.5)$ \\
\hline & $N=37^{a}$ & \\
\hline \multirow[t]{2}{*}{ FBI-ALS } & $9.7(9.9)$ & $9.3(9.1)$ \\
\hline & $\mathrm{N}=36^{\mathrm{a}}$ & \\
\hline
\end{tabular}

Abbreviations: ALS-CBS $=$ Amyotrophic Lateral Sclerosis-Cognitive Behavioral Screen CNS-LS = Center for Neurologic Study-Lability Scale; COWAT = Controlled Oral Word Association Test; FBI-ALS = Frontal Behavioral Inventory-ALS; PLS = primary lateral sclerosis; SSEP = somatosensory evoked potential.

All data are mean (SD) unless otherwise indicated.

${ }^{a}$ Number of patients with complete cognitive testing data.

${ }^{b}$ Percentages derived from the Written Verbal Fluency Index or the COWAT (consisting of 3 scores)-see the text for further explanation.

patients with complete data (figure 2). The first branch separated 1 patient (case ID 7), leaving 24. The second branch separated 6 patients, with 18 remaining. The resulting 2 clusters differentiated the 6 patients separated in the second step from the remaining patients (i.e., case ID 7 was grouped with the 18 for a total of 19 patients in that cluster).

The Lasso model selected 6 of the 19 variables associated with the cluster groupings: dysarthria, dysphagia, objective bulbar signs, urinary urgency, weight loss, and sensory impairment, the presence of which characterized the smaller cluster of $6 \mathrm{pa}$ tients. Some of these clinical features were also present in other patients. None of these 6 variables was statistically significant in a joint logistic regression model, which predicted cluster membership. However, in individual logistic regression models, dysphagia $(p=0.01)$, objective bulbar signs $(p=0.01)$, and urinary urgency $(p=0.002)$ were associated with cluster membership. The modeling step was not based on an a priori specification of clustering variables and was exploratory in nature; therefore, the $p$ values should be interpreted cautiously.

Secondary analyses. Using all 41 patients, the plot of the within-groups sum of squares by number of clusters was similar to the curve found in the primary analysis, without an obvious bend. We again proceeded with the assumption of 2 clusters for ease of interpretation. Similar to the primary analysis, the Lasso model selected 7 of the 18 variables associated with the grouping: dysarthria, dysphagia, objective bulbar signs, minimum EMG changes, urinary urgency, weight loss, and female sex. Of these 7 variables, none were associated with cluster membership in a joint logistic regression model. However, in individual logistic regression models, dysphagia $(p=0.004)$, objective bulbar signs $(p<0.001)$, urinary urgency $(p<0.001)$, and female sex $(p=0.02)$ were associated with cluster membership. With the exception of sex, the results confirmed the primary analysis. Finally, we tested for cluster differences in all the cognitive variables that were not included in the $\mathrm{k}$-means clustering and none differed across the 2 groups.

We repeated the analyses for the 37 patients with complete data for the Amyotrophic Lateral SclerosisCognitive Behavioral Screen (ALS-CBS) variables. The plot of the within-groups sum of squares by number of clusters was similar to that shown in figure 1. We proceeded assuming 2 clusters. The Lasso model selected 9 of the 18 variables associated with the grouping: age, disease duration at baseline, dysarthria, dysphagia, objective bulbar signs, minimum EMG changes, urinary urgency, sensory impairment, and female sex. Of these 9 variables, none were associated with cluster membership in a joint logistic model. However, in individual logistic regression models, dysphagia ( $p=0.009$ ), objective bulbar signs $(p<0.001)$, urinary urgency $(p=0.004)$, and female sex $(p=0.009)$ were associated with cluster membership. The final analysis used patients with no missing values on the ALS-CBS cognitive and behavior scales. Results (not shown) were similar to those in the primary analysis.

Exome sequencing. Table e- 1 lists the mutations detected by C9ORF72 expansion testing and exome sequencing in genes associated with HSP, familial 
Figure 1 Within-groups sum of squares vs number of clusters

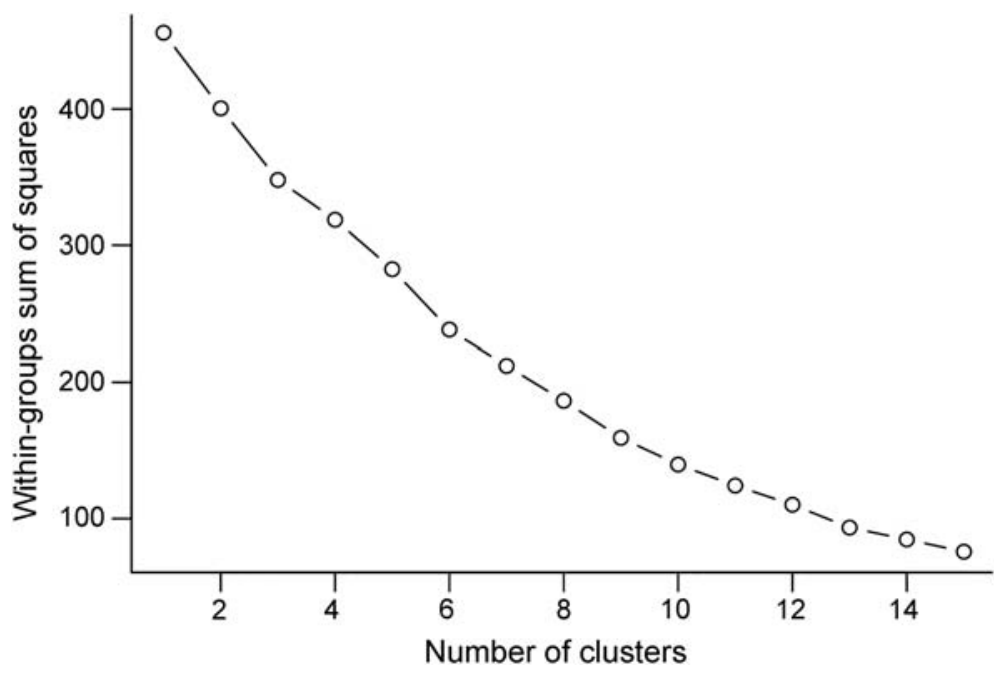

Within-groups sum of squares vs number of clusters to determine the number needed for k-means cluster analysis. The cluster algorithm assumes a given number of clusters to determine the grouping of patients. Figure 1 shows 15 separate cluster algorithms with 1-15 clusters assumed and the within-groups sum of squares calculated for each. Optimally, one would like to select a small number of clusters for ease of interpretation but with a small within-groups sum of squares. When there is a bend (like an elbow), it provides evidence that the gain is large (i.e., reducing the sum of squares) in identifying a fixed number of clusters with decreased evidence to increase the number of clusters. Our analysis showed no evidence of a bend, so we chose 2 clusters for convenience of interpretation.

ALS, and other known neurologic diseases. We identified one previously described pathogenic mutation in the SPG7 gene in heterozygous form (A510V) in patient $1^{21}$ and one C9ORF72 expansion in patient $8 .{ }^{14}$ Patient 1 and patient 8 belong to the larger cluster (figure 2). Patient 11 had a predicted pathogenic heterozygous mutation in $P A R K 2,{ }^{22}$ and patient 41 had a predicted risk allele in DCTN1. ${ }^{23}$

Patient 11 also belonged to the larger cluster; patient 41 was not clustered because cognitive testing data were incomplete. A predicted pathogenic mutation in the SYNE2 gene was identified in patient $6 .^{24}$ However, there was no evidence of myopathy. Several other patients showed SNPs of unknown significance in the PARK2 (patient 23), VEGFA (patient 26), CLNG (patient 29), BTD (patient 29), and LRKK2 (patient 32) genes.

From our exome sequencing performed on all patients with PLS, we have identified a combined 4,500 rare (population frequency less than $1 \%$ ) missense or nonsense variants that occurred in at least 2 patients with PLS. Among these 4,500 variants, one-third mapped to genes with some disease-related annotation in the Online Mendelian Inheritance in Man database, and about one-third were predicted to disrupt protein function based on functional prediction algorithms (SIFT, Provean). We are currently refining our analysis and classification of these variants.

DISCUSSION Our overall goal was to take the first step toward determining, through analyzing phenotypes and genotypes, whether PLS is a single entity or a syndrome including multiple neurodegenerative variants. The expanding knowledge of genotypic expression has made it clear that the traditional view of the relationship between phenotypes and genotypes is simplistic. Detailed phenotypic information is now considered essential to understanding new or poorly defined diseases, such as PLS. We accomplished our goal in 2 ways: first, by using k-means clustering of phenotypic characteristics to identify groups of similar patients, and second, by using exome sequencing to identify possible genes associated with these phenotypes.

Our study has several strengths. We prospectively enrolled only those patients with clinically "definite"

Figure 2 Cluster dendrogram

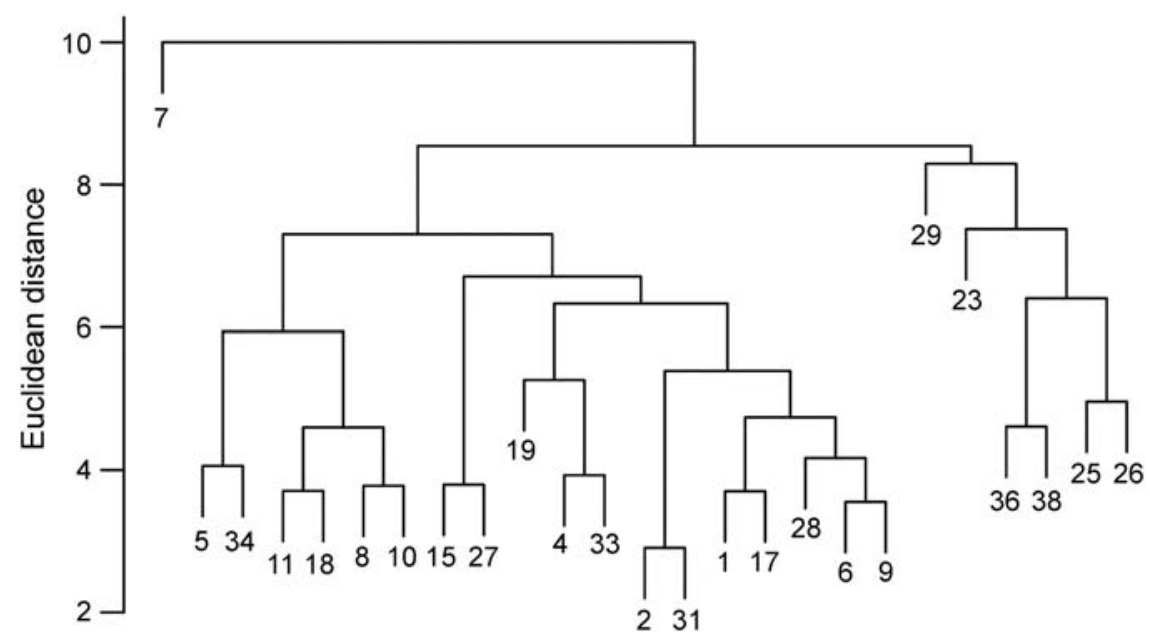

Cluster dendrogram for similarity between patients with primary lateral sclerosis with complete datasets $(\mathrm{N}=25)$. Each patient is denoted by a corresponding code number. 
PLS to narrow clinical diversity and sought to identify any further possible subsets using phenotype cluster analyses. Subsequent k-means clustering, combined with a final model-building step, suggests that patients with PLS may be classified into 2 groups based on the presence or absence of the following clinical variables: dysarthria, dysphagia, objective bulbar signs, urinary urgency, weight loss, and sensory impairment. One limitation of this approach, however, is that the Lasso method chooses variables randomly from sets of highly correlated variables; thus, the selected variables may represent a subset of the entire group of variables. ${ }^{25}$ However, the results of sensitivity analyses were essentially similar, and these put fewer restrictions on the number of variables with complete data. Therefore, our results suggest that the diagnosis of PLS may represent at least 2 different groups of patients.

Another important strength is genetic analysis of all patients to test for the presence of known diseasecausing mutations associated with other neurodegenerative disorders. Mutations in SPG7 (A510V) result in autosomal recessive spastic paraplegia. The mutation found in patient 1 is a heterozygous SPG variant, which is reported to predispose individuals to lateonset, complex neurodegenerative disorders. ${ }^{26} \mathrm{~A}$ more recent study indicates that this particular heterozygosity can be pathogenic for $\mathrm{HSP}^{27}$ (personal communication with Dr. John Fink, University of Michigan, June 2014). Patient 8 presented with clinically typical PLS but was found to have C9ORF72 hexonucleotide repeat expansion. A Dutch group previously described a single patient with C9ORF72 expansion in a PLS cohort of 110 patients. ${ }^{28}$ Although C9ORF72 expansion is predominantly found in patients with ALS or ALS plus frontotemporal dementia, it has been described in other neurodegenerative diseases. ${ }^{28}$ Variable clinical phenotypes may be the result of complex environmental interactions and genetic modifiers. ${ }^{29}$ Our study and the Dutch study indicate that C9ORF72 expansion can be expressed phenotypically as typical PLS.

Features of atypical Parkinson disease (PD) have been reported in patients with a diagnosis of PLS. ${ }^{30,31}$ Although none of our patients had clinical features of $\mathrm{PD}$, we found mutations in the PARK2 gene (Arg275Trp) previously described as a cause of familial PD. This PARK2 gene mutation is pathogenic when homozygous, but the heterozygous variants are reported to affect PD onset ${ }^{32}$ and phenotype ${ }^{33,34}$ The LRRK2 mutation is known to cause autosomal dominant familial PD, although in one study of large families the R1514Q mutation in our patient (patient 32) had not segregated with PD. ${ }^{35}$ Yet other LRRK2 mutations are found in sporadic PD cases, ${ }^{36}$ and typical MND is reported in patients with familial PD resulting from a different LRRK2 mutation. ${ }^{37}$ Although the LRRK2 mutation is interesting, its pathogenic significance in our patient with PLS is at best uncertain, and thus we consider it to be an SNP at this time. Our exome sequencing also identified another important mutation that is a known pathogenic mutation of the DCTN1 gene $^{38}$; although this mutation is rare, it has been reported in an apparent sporadic case of ALS. ${ }^{22}$

Therefore, exome sequencing identified 4 potentially pathogenic mutations occurring in 34 patients with PLS (nearly 12\%), all associated with neurodegenerative diseases such as ALS, HSP, and PD. With increasing knowledge in the near future, we may need to consider genetic counseling for patients who are found to have known gene mutations. Patients with 2 of these mutations belong to the cluster having the larger number of patients. Five other SNPs of unknown clinical significance were also detected (PARK2, $V E G F A, C N L 6, B T D$, and $L R R K 2)$ as well as a predicted pathogenic mutation in SYNE2. Based on our experience, we recommend that patients with a diagnosis of PLS, especially those participating in research studies, be screened for pathogenic mutations using partial or complete exome sequencing to determine the prevalence of known pathogenic mutations and the pathogenic significance of variants of unknown significance. Further studies are needed to clarify the significance of these SNPs and the SYNE2 mutation.

The diagnosis of PLS is still problematic because of the time required to wait before making a diagnosis and how strictly pure UMN involvement is defined. We decided to use very strict criteria (5 years after symptom onset before enrollment) so we could investigate clinically well-defined PLS cases. Yet waiting for such an extended time to make the diagnosis is neither practical nor helpful because physicians need to make a diagnosis at the earliest opportunity. Investigators also want to start PLS research at earlier stages of the disease, when active biological changes can be more readily found. We made every effort to select patients who had a "definite" diagnosis of PLS, short of autopsy proof. Therefore, disease diagnosed using our clinical criteria as described above (pure UMN dysfunction with normal EMG along with bulbar symptom(s), normal neuroimaging, and all definable diseases excluded) with negative exome sequencing and a negative C9ORF72 expansion test can be considered clinically "definite" PLS for research purposes currently. PLS with a shorter disease duration (3-4 years) could be considered clinically "probable" or clinically "possible." These clinically probable or possible cases could be elevated to definite PLS if the exome sequence and C9ORF72 expansion tests detect no known molecular abnormalities. Until we have improved biological or genetic markers, such criteria may be suitable for research investigation of PLS. We need an updated international consensus for PLS diagnosis. 
Study limitations. Although the number of patients we studied is not small in comparison to other studies in PLS, a larger number of patients is required to achieve definitive results for both clinical clustering and exome sequencing analyses. We need to include more centers, preferably with long-term funding to allow adequate time for recruitment and to overcome the limitations involved with studying such a rare disease. Another limitation is the selection of patients with clinically "definite" PLS, which included patients who are in more advanced stages of the disease. Waiting a long period of time to diagnose PLS is a major drawback. Ideally, we should study PLS much earlier in the disease course, when biomarkers that can identify early PLS are most essential. To find such biomarkers, studying patients with clinically "definite" PLS is a necessary step.

We identified 2 phenotypic groups and a small number of patients with gene mutations known to be pathogenic for various neurodegenerative diseases. Further studies are needed. Our study is only the beginning of such investigations in PLS, providing a foundation that will hopefully stimulate other investigators to examine and expand our phenotypic and genetic studies in order to more fully understand PLS.

\section{AUTHOR CONTRIBUTIONS}

Dr. Mitsumoto served as the primary investigator for this study. He was involved in all stages of this project, including data collection, analyses, and preparing the draft of the manuscript. Dr. Nagy assisted in analyses of biomarkers in addition to reviewing the manuscript. Dr. Gennings assisted in statistical analyses, specifically cluster analyses, in addition to reviewing the manuscript. Dr. Murphy assisted in cognitive analyses in addition to reviewing the manuscript. Dr. Andrews assisted in data management for the entire study in addition to reviewing the manuscript. Dr. Goetz assisted in general statistical analyses in addition to reviewing the manuscript. Dr. Floeter served as a site investigator for National Institute of Neurological Diseases and Stroke and was involved in planning the study, data collection, and reviewing the manuscript. Mr. Hupf assisted in planning and coordination of the study, data collection, and reviewing the manuscript. Ms. Singleton assisted in coordination of the study, data collection, and reviewing the manuscript. Dr. Barohn served as site investigator for the University of Kansas and was involved with planning of the study, data collection, and reviewing the manuscript. Dr. Nations served as site investigator for University of Texas Southwestern Medical Center and was involved with planning of the study, data collection, and reviewing the manuscript. Dr. Shoesmith served as site investigator for Western University, London, Ontario, Canada, and was involved with planning of the study, data collection, and reviewing the manuscript. Dr. Kasarskis served as site investigator for University of Kentucky and was involved with planning the study, data collection, and reviewing the manuscript. Dr. Factor-Litvak assisted as the co-PI and was involved with the planning and statistical analyses as well as analyses of biomarkers, exposures, and cognition. She also reviewed the manuscript. All PLS COSMOS Study Group members assisted in data collection and reviewing the manuscript.

\section{ACKNOWLEDGMENT}

The authors appreciate and thank the patients and families who participated in this study. John Fink, MD, Department of Neurology, University of Michigan, kindly gave suggestions and provided unpublished information. Jennifer Langsdorf, MD, Peripheral Neuropathy Center, Weill-Cornell University, gave a follow-up examination and performed EMG test for our study. Cassandra Talerico, $\mathrm{PhD}$, provided substantive editing and comments. Georgia Christodoulou, MA, assisted with the preparation of the manuscript, including some copyediting and preparing materials for submission (neither met the criteria for authorship).

\section{STUDY FUNDING}

The study was funded by Spastic Paraplegia Foundation (SPF), MDA Wings Over Wall Street, NIEHS (R01ES016348) for the ALS COSMOS study, and private donations from Mr. and Mrs. David Marren.

\section{DISCLOSURE}

Dr. Mitsumoto has served on scientific advisory boards for Avanir, Knopp, Sanofi-Aventis, the Japanese Society of Neurology, and Biogen and received research support from SPF, MDA Wings Over Wall Street, NIEHS (R01ES016348), Avanir, Teva, Knopp, Columbia, SanofiAventis, Biogen, National Institute of Neurological Disorders and Stroke, NIEHS, ATSDR, and private donations from Mr. and Mrs. David Marren. Dr. Nagy and Dr. Gennings report no disclosures. Dr. Murphy is employed by and has received travel funding and/or speaker honoraria from INC Research. Dr. Andrews has consulted for the Research Triangle Institute and Michael J. Fox Foundation and has received research support from Columbia University and the Research Foundation for Mental Hygiene. Dr. Goetz reports no disclosures. Dr. Floeter has served on the editorial board of Muscle and Nerve and is employed by National Institute of Neurological Disorders and Stroke/NIH. Mr. Hupf has received research support from NIEHS, Spastic Paraplegia Foundation, and Muscular Dystrophy Association Wings Over Wall Street. Ms. Singleton reports no disclosures. Dr. Barohn has served on a scientific advisory board for Diaphragm Stem Study; has received funding for travel and/or speaker honoraria from Grifols, NuFactor, KU, and Walgreens; has served on the editorial board of the Journal of Clinical Neuromuscular Disease; has served on speakers' bureaus for Grifols, Baxter, and Sanofi/ Genzyme; and has received research support from Cytokinetics, GSK, CSL-Berhring, Alexion, Sanofi/Genzyme, Biomarin, PTC, Eli Lilly, NIH/National Institute of Neurological Disorders and Stroke, and FDA. Dr. Nations has served on Grifols' speakers' bureau; has received funding for travel and/or speaker honoraria from Grifols; and has received research support from Novartis, Alexion, FDA, and NIH-National Institute of Neurological Disorders and Stroke. Dr. Shoesmith reports no disclosures. Dr. Kasarskis serves as Associate Editor of Amyotrophic Lateral Sclerosis and Frontotemporal Degeneration; has consulted for ASubio Pharmaceuticals, Cytokinetics Pharmaceuticals, and Neuraltus Pharmaceuticals; and has received research support from Neuraltus Pharmaceuticals, Cytokinetics, National Institute of Neurological Disorders and Stroke, NIEHS, and the ALS Association. Dr. Factor-Litvak has received funding for travel and/or speaker honoraria from the Environment and Health Fund; serves on the editorial boards of the Journal of Environmental and Public Health and Neurotoxicology; and has received research support from the Columbia Global Center/FABERJ, NIEHS, CDC, National Institute of Mental Health, Institute of Child Health and Development, Environmental Health Fund, National Institute of Diabetes, Digestive Disorders and Kidney Disorders, National Cancer Institute/National Institute of Environmental Health Sciences, and National Institute of Environmental Health Science/Environmental Protection Agency. Go to Neurology.org/ng for full disclosure forms.

Received February 9, 2015. Accepted in final form March 17, 2015.

\section{REFERENCES}

1. Le Forestier N, Maisonobe T, Spelle L, et al. Primary lateral sclerosis: further clarification. J Neurol Sci 2001; 185:95-100.

2. D'Amico E, Pasmantier M, Lee YW, Weimer L, Mitsumoto H. Clinical evolution of pure upper motor neuron disease/dysfunction (PUMMD). Muscle Nerve 2013;47:28-32.

3. Gordon PH, Cheng B, Katz IB, Mitsumoto H, Rowland LP. Clinical features that distinguish PLS, upper 
motor neuron-dominant ALS, and typical ALS. Neurology 2009;72:1948-1952.

4. Pringle CE, Hudson AJ, Munoz DG, Kiernan JA, Brown WF, Ebers GC. Primary lateral sclerosis-clinical features, neuropathology and diagnostic criteria. Brain 1992;115:495-520.

5. Tartaglia MC, Rowe A, Findlater K, Orange JB, Grace G, Strong MJ. Differentiation between primary lateral sclerosis and amyotrophic lateral sclerosis -examination of symptoms and signs at disease onset and during follow-up. Arch Neurol 2007;64:232-236.

6. Le Forestier N, Maisonobe T, Piquard A, et al. Does primary lateral sclerosis exist? A study of 20 patients and a review of the literature. Brain 2001;124: 1989-1999.

7. Zhai P, Pagan F, Statland J, Butman JA, Floeter MK. Primary lateral sclerosis: a heterogeneous disorder composed of different subtypes? Neurology 2003;60:1258-1265.

8. Mitsumoto H, Factor-Litvak P, Andrews H, et al. ALS Multicenter Cohort Study of Oxidative Stress (ALS COSMOS): study methodology, recruitment, and baseline demographic and disease characteristics. Amyotroph Lateral Scler Frontotemporal Degener 2014; 15:192-203.

9. Chan S, Kaufmann P, Shungu DC, Mitsumoto H. Amyotrophic lateral sclerosis and primary lateral sclerosis: evidence-based diagnostic evaluation of the upper motor neuron. Neuroimaging Clin N Am 2003;13:307-326.

10. Brugman F, Veldink JH, Franssen H, et al. Differentiation of hereditary spastic paraparesis from primary lateral sclerosis in sporadic adult-onset upper motor neuron syndromes. Arch Neurol 2009;66:509-514.

11. Lezak MD. Neurological Assessment. New York: Oxford University Press; 2004.

12. Tanioka $\mathrm{K}$, Tadohisa H. Effect of data standardization on the result of k-means clustering. In: Wolfgang G, Geyer-Schulz A, Schmidt-Thieme L, Kunze J, editors. Challenges at the Interface of Data Analysis, Computer Science, and Optimization, Studies in Classification, Data Analysis, and Knowledge Organization. Berlin: Springer; 2012:59-67.

13. Tibshirani R. Regression shrinkage and selection via the Lasso. J Roy Stat Soc B Met 1996;58:267-288.

14. Renton AE, Majounie E, Waite A, et al. A hexanucleotide repeat expansion in C9ORF72 is the cause of chromosome 9p21-linked ALS-FTD. Neuron 2011;72:257-268.

15. Chin EL, da Silva C, Hegde M. Assessment of clinical analytical sensitivity and specificity of next-generation sequencing for detection of simple and complex mutations. BMC Genet 2013;14:6.

16. Landrum MJ, Lee JM, Riley GR, et al. ClinVar: public archive of relationships among sequence variation and human phenotype. Nucleic Acids Res 2014;42: D980-D985.

17. 1000 Genomes Project Consortium, Abecasis GR, Altschuler D, et al. A map of human genome variation from population-scale sequencing. Nature 2010;467:1061-1073.

18. Krumm N, Sudmant PH, Ko A, et al. Copy number variation detection and genotyping from exome sequence data. Genome Res 2012;22:1525-1532.

19. Luu TD, Rusu AM, Walter V, et al. MSV3d: database of human MisSense variants mapped to 3D protein structure. Database (Oxford) 2012:bas018.

20. Choi Y, Sims GE, Murphy S, Miller JR, Chan A. Predicting the functional effect of amino acid substitutions and indels. PLoS One 2012;7:e46688.
21. Roxburgh RH, Marquis-Nicholson R, Ashton F, et al. The p.Ala510Val mutation in the SPG7 (paraplegin) gene is the most common mutation causing adult onset neurogenetic disease in patients of British ancestry. J Neurol 2013;260:1286-1294.

22. Cookson MR, Lockhart PJ, McLendon C, O'Farrell C, Schlossmacher M, Farrer MJ. RING finger 1 mutations in Parkin produce altered localization of the protein. Hum Mol Genet 2003;12:2957-2965.

23. Münch C, Sedlmeier R, Meyer T, et al. Point mutations of the p150 subunit of dynactin (DCTN1) gene in ALS. Neurology 2004;63:724-726.

24. Zhang QP, Bethmann C, Worth NF, et al. Nesprin-1 and -2 are involved in the pathogenesis of Emery Dreifuss muscular dystrophy and are critical for nuclear envelope integrity. Hum Mol Genet 2007;16:2816-2833.

25. Zou H, Hastie T. Regularization and variable selection via the elastic net. J Roy Stat Soc B 2005;67:301-320.

26. Klebe S, Depienne C, Gerber S, et al. Spastic paraplegia gene 7 in patients with spasticity and/or optic neuropathy. Brain 2012;135:2980-2993.

27. Sanchez-Ferrero E, Coto E, Beetz C, et al. SPG7 mutational screening in spastic paraplegia patients supports a dominant effect for some mutations and a pathogenic role for p.A510V. Clin Genet 2013;83:257-262.

28. van Rheenen W, van Blitterswijk M, Huisman $\mathrm{MH}$, et al. Hexanucleotide repeat expansions in C9ORF72 in the spectrum of motor neuron diseases. Neurology 2012;79: 878-882.

29. Cooper-Knock J, Shaw PJ, Kirby J. The widening spectrum of C9ORF72-related disease; genotype/phenotype correlations and potential modifiers of clinical phenotype. Acta Neuropathol 2014;127:333-345.

30. Gilbert RM, Fahn S, Mitsumoto H, Rowland LP. Parkinsonism and motor neuron diseases: twenty-seven patients with diverse overlap syndromes. Mov Disord 2010;25:1868-1875.

31. Norlinah IM, Bhatia KP, Ostergaard K, Howard R, Arabia G, Quinn NP. Primary lateral sclerosis mimicking atypical parkinsonism. Mov Disord 2007;22:2057-2062.

32. Sun M, Latourelle JC, Wooten GF, et al. Influence of heterozygosity for parkin mutation on onset age in familial Parkinson disease: the GenePD study. Arch Neurol 2006; 63:826-832.

33. Farrer M, Chan P, Chen R, et al. Lewy bodies and parkinsonism in families with parkin mutations. Ann Neurol 2001;50:293-300.

34. Klein C, Lohmann-Hedrich K, Rogaeva E, Schlossmacher MG, Lang AE. Deciphering the role of heterozygous mutations in genes associated with parkinsonism. Lancet Neurol 2007;6: 652-662.

35. Nichols WC, Marek DK, Pauciulo MW, et al. R1514Q substitution in Lrrk2 is not a pathogenic Parkinson's disease mutation. Mov Disord 2007;22:254-257.

36. Anfossi M, Colao R, Gallo M, et al. Identification of three novel LRRK2 mutations associated with Parkinson's disease in a Calabrian population. J Alzheimers Dis 2014;38: 351-357.

37. Whittle AJ, Ross OA, Naini A, et al. Pathogenic Lrrk2 substitutions and amyotrophic lateral sclerosis. J Neural Transm 2007;114:327-329.

38. Vilarino-Guell C, Wider C, Soto-Ortolaza AI, et al. Characterization of DCTN1 genetic variability in neurodegeneration. Neurology 2009;72:2024-2028. 


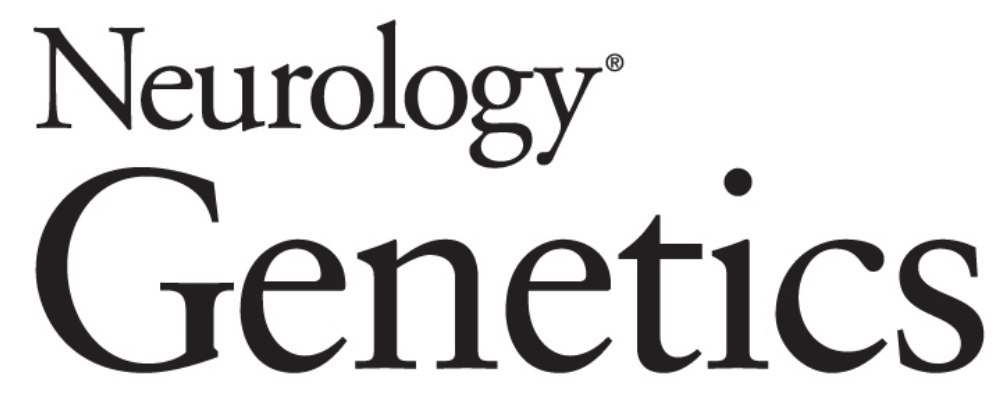

\section{Phenotypic and molecular analyses of primary lateral sclerosis Hiroshi Mitsumoto, Peter L. Nagy, Chris Gennings, et al. Neurol Genet 2015;1; \\ DOI 10.1212/01.NXG.0000464294.88607.dd}

\section{This information is current as of April 16, 2015}

\section{Updated Information \& Services \\ including high resolution figures, can be found at: http://ng.neurology.org/content/1/1/e3.full.html \\ Supplementary Material \\ Supplementary material can be found at: http://ng.neurology.org/content/suppl/2015/04/16/1.1.e3.DC1 http://ng.neurology.org/content/suppl/2015/04/16/1.1.e3.DC2 \\ References \\ This article cites 35 articles, 1 of which you can access for free at: http://ng.neurology.org/content/1/1/e3.full.html\#\#ref-list-1 \\ Citations \\ This article has been cited by 8 HighWire-hosted articles: http://ng.neurology.org/content/1/1/e3.full.html\#\#otherarticles \\ Permissions \& Licensing its entirety can be found online at: http://ng.neurology.org/misc/about.xhtml\#permissions \\ Reprints \\ Information about ordering reprints can be found online: http://ng.neurology.org/misc/addir.xhtml\#reprintsus} Information about reproducing this article in parts (figures,tables) or in

Neurol Genet is an official journal of the American Academy of Neurology. Published since April 2015, it is an open-access, online-only, continuous publication journal. Copyright $(2015$ American Academy of Neurology. All rights reserved. Online ISSN: 2376-7839.

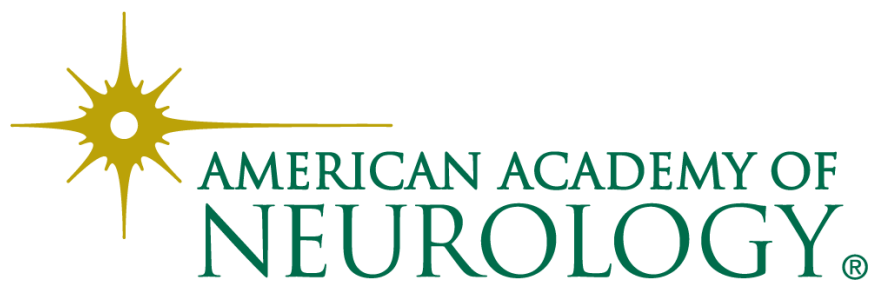

